\title{
The Application of EDA in the Electronic Technology Practice Course in the Higher Vocational Colleges
}

\author{
1, aWANG Xiaohua \\ ${ }^{1}$ Institute of Applied Electronics, Chongqing College of Electronic Engineering, Chongqing, \\ 401331
}

a12934236@qq.com

Keywords: Application, EDA, Electronic Technology Practice Course, Higher Vocational Colleges

\begin{abstract}
With the rapid development of electronic information technology, it has created the conditions for the teaching and practice of electronic technology in vocational colleges. The most outstanding case is the application of EDA technology in the course of electronic technology practice. The practice shows that: the using of EDA technology in teaching can help achieve the teaching goal and it is conducive to students' mastering of modern electronic system design techniques and to promote the practical training of highly skilled personnel.
\end{abstract}

\section{Introduction}

Higher vocational education through the training of skilled personnel, combined with economic and social development, take the initiative to serve the local economic development, in order to boost the local economy and its sustainable development. In recent years, in the course of construction and development of vocational colleges, with particular emphasis on connotation construction and training conditions to the characteristics of running a school, to the quality of legislation school. However, in the construction of training conditions, the vocational colleges are almost all in the allocation of equipment on the pursuit of practical, practical, advanced enough to make efforts, however, we must clearly recognize that our training conditions Construction, there is no end, we cannot all suitable for professional development and skills training needs of the hardware facilities continue to buy, cannot be in the school to master all the job skills, therefore, to really pay attention to the combination to solve the shortage of hardware and job docking training requirements, and the school experiment should consider the necessary, sufficient, practical, Economic, reliable, advanced for the degree, there are many ways to solve, for many years in science and engineering colleges vocational education in the use of verification experiments, is still active in the teaching of vocational training activities, this type of experiment It is the knowledge verification work of skill training, especially in the fields of electrical engineering, electrical engineering, electrical engineering, electrical engineering, electrical engineering, electrical engineering, electrical engineering and so on. In the experiment, the proportion of resources (equipment, consumables and teaching expense) According to the incomplete statistics, the electrical and electronic experiments and the electronic experiment have been carried out in a large number of colleges and universities each year. The consumption of resources is about 1/6-1/3 of the consumption of all the verification experiments. In this paper, EDA simulation technology is used in the experiment of electronic and electrical engineering in vocational colleges.

\section{The Overview of EDA}

EDA (Electronic Design Automation) refers to the computer as the working platform, integration of application of electronic technology, computer technology, intelligent technology and the latest achievements of the development of common electronic CAD software package. Mainly to assist in three aspects of the design work, both IC design, electronic circuit design and PCB design. EDA technology has gone through three stages of development. EDA technology represents the latest development of today's electronic design technology. It not only provides electronic technology designers with top-down design concept, but also provides an extremely convenient and scientific 
experimental teaching platform for teaching. Electrical and electronic courses in the electrical foundation, analog electronic technology and digital electronic technology can be EDA simulation software, the circuit diagram of the drawing, design, simulation and analysis. It should be said that EDA simulation software will be applied to the electrical, electronic courses is a means of teaching innovation, but also improve the quality of teaching the preferred method. Through the use of EDA technology software to support the theory of electronic technology courses, students can strengthen the basic knowledge of circuit theory to master; improve students' theory and practice of combining the comprehensive ability; through the completion of integrated circuit design debugging hard experiment to develop students' practical hands. Practice has proved that this combination of theory and practice, soft experimental and hard experimental combination of training for students to cultivate students' awareness of innovation and improve the comprehensive design ability of students is very useful.

\section{The Basic Function of EDA Simulation Technology}

Electrical and electronic courses are designed to require students to master all types of electrical and electronic devices, drawings and drawings of the circuit diagram and the working principle of the circuit, and can master the analysis of the circuit. Its function is as follows:

EDA software has established various types of component design database module. It includes: power source library, basic component library, diode library, transistor library, analog integrated device library, TTL digital device library, CMOS device library, other digital device library, mixed device library, indicating device library, hybrid device library, Library, mechanical and electrical equipment such as libraries. Rich library of components for students to understand all kinds of electricians, electronic components pave the way for a solid foundation, but also through the device library to understand the performance of various devices and parameters, and can provide innovative design and the use of the endless any economic burden of test elements.

EDA software components can be created and edited. You can edit, modify and create new components for your new devices. This function provides a better technology platform for students' independent innovation. Therefore, making full use of EDA technology teaching is a good way to improve students' innovative thinking teaching.

EDA software has circuit schematic design of the input sub-module. Through this function can complete the composition of various components of the circuit schematic. Through the schematic design can help students understand the structure of the schematic diagram and the relationship between the circuit at all levels, the students read the map and map to play a multiplier effect.

EDA software integrated simulation module configuration such as ammeter, voltmeter, function signal generator, oscilloscope, power meter, sweep meter, word signal generator, logic analyzer, logic converter, distortion analyzer, Spectrum analyzers and other instruments. They provide simulation of various types of analog circuits, dynamic voltage, current parameters and waveform analysis. On the digital logic circuit can test the truth of the gate circuit and analysis of the gate of the time waveform.

Multiple types of simulation analysis such as DC operating point analysis, AC analysis, transient analysis, Fourier analysis, noise analysis, distortion analysis, DC sweep analysis, temperature scanning analysis, parametric scanning analysis, sensitivity analysis, transfer function analysis, pole-zero analysis Wait. The analysis results are displayed intuitively by numerical value or waveform, which provides rich and real data for the analysis of the circuit, so that the conclusion can meet the theoretical value and practicality.

\section{The Role of EDA Technology in the Electronic Technology Practice Course}

With the rapid development of electronic technology, the application of EDA has penetrated into various fields, which undoubtedly put a higher demand for our teaching, we need educators to train familiar with the electrical and electronic technology skills so that they should have a strong theoretical knowledge and skilled operating skills, through effective testing skills. 
Through the EDA course learning, can well consolidate and expand the electronic technology knowledge, realize knowledge to ability transformation. Solid grasp of the basic theoretical knowledge of teaching materials, so that students' deeper understanding of the general conclusion of the reasoning more clear understanding of the changes in the electrical system more practical, EDA through a lot of simulation experiments and practical operation can achieve this purpose.

The strengthening of experimental courses can improve the practical skills of students. Higher vocational college students must be able to quickly adapt to the first line of the production line of job skills, with strong practical skills and the courage to reform and innovation. But also skilled talent in the market competition must have the conditions, so do a good job of experimental teaching and students to create an atmosphere of imagination to play the space is necessary. The practice of EDA simulation teaching proves that its application only needs to mobilize the interest and enthusiasm of the students, without increasing the expenditure of teaching expendable materials. This method is conducive to improving the quality of personnel training.

It is conducive to training students' good occupation habits, professional ethics and psychological quality. With good professional habits, professional ethics and psychological quality of graduates in the future work with competitive ability is one of the important conditions. And good professional habits, professional ethics and psychological stability is to develop on a regular basis. Experiment teaching is an important way to cultivate this quality. Because of the rationality of the experimental process, rigorous, scientific, not tolerate the slightest hypocrisy. The success and failure of the experiment can also gradually improve the psychological quality of students in the face of failure.

EDA simulation is conducive to cultivating more people to adapt to new technical requirements. The function of higher vocational education has become the service for the learner, the service for the employer and the social economic development. The demand for talent changes, the rapid development of technology is the need for educators have a sense of advancement of personnel training. This awareness must be scientific, new, fast, or even jumping. In particular, the cultivation of talents in vocational colleges needs to master the new technology of professional teachers, but also a new technology equipment to meet the requirements of personnel training. New technologies, new equipment, more and less investment will directly affect the talent into the talent market, the most cutting-edge opportunities. This requires us to think innovation, teaching means innovation. Change the traditional idea, establish EDA consciousness, take EDA simulation technology as the breakthrough point and introduce the computer-assisted teaching method. Further development will also look forward to the maturity of the network virtualization experimental system to replace the original teaching equipment, improve the quality of teaching, teaching practice to solve practical problems, thereby speeding up the cultivation of high-quality personnel.

It is conducive to the cultivation of high-quality teaching staff, and further promote the reform of electronic technology courses with the rapid development of electronic technology, on the one hand the community to improve the requirements of electronic technology talent, on the other hand, teachers cannot adapt to the original knowledge Social development needs. This requires our teachers to first improve their knowledge and technical ability, by sending relevant teachers to the domestic universities to study, learning and other learning to improve, but also by holding EDA technical seminars, short courses, etc. to achieve the updated teachers And expand the horizons and update the knowledge of the purpose, prompting teachers to actively participate in teaching reform, reform teaching content, to participate in the preparation of new textbooks, innovation consciousness into the curriculum construction. Only the concept of teachers has changed, knowledge is updated, teaching reform will be able to implement.

\section{The Status Quo of EDA in the Experimental Teaching}

EDA technology is to adapt to the requirements of modern electronic product design to absorb the latest achievements of multidisciplinary and the formation of a new technology, as a high-level vocational and technical, high-speed, low- EDA institutions will be applied to the experimental teaching of electronic technology is still more effective, and can produce good experimental results, 
and to fill the school laboratory equipment and lack of space defects.

Basic skills training: basic circuit simulation, digital circuit analysis, circuit optimization analysis, the stage is the primary stage of practice, the main training students master the basic operational skills for theoretical teaching to establish perceptual knowledge, and lay a good foundation. The purpose is to enable students to use computers and EDA software in-depth study and understanding of basic knowledge of electronic technology, the establishment of basic knowledge of electronic circuit system to master the circuit system analysis method, through the circuit characteristic curve and data to analyze the function and characteristics of the circuit and complete Principle - based Verification Experiment in Traditional Experimental. Using the "EDA software platform" can be easily carried out circuit AC and DC analysis, frequency response analysis, transient analysis, temperature scanning analysis and noise analysis. The advantage of using EDA software is that the difficulties in teaching can be explained by graph and curve, which reduces the difficulty of mathematics, but vividly illustrates the basic theory and concept of circuit system. Students can use the software to provide a wealth of virtual electronic components and instruments for complex circuit design and analysis, the design of the circuit from the laboratory components of the resource constraints. At the same time these software resources in the educational resources is very easy to download and upgrade, in the PC machine can run. Students in their spare time according to their own interests to learn and complete the design, so that both can fully mobilize the enthusiasm of students to learn, but also can reduce the experimental funds and teachers to prepare the laboratory time in the current university funding shortage and lack of real reality, After all, is a good method.

Professional skills training: through the creation of "programmable logic device", "VHDL language and digital system design" EDA technology courses, learning VHDL language, Verilog language EDA development tools, learning hardware description language, state machine and Circuit schematics and other methods in the FPGA / CPLD chip design integrated electronic applications. Students have taken basic courses in electronic technology, including analog and digital circuits, and have been trained in the design of electronic technology courses. They have acquired the basic knowledge and skills of electronic technology required for electronic system design. By learning to master the advanced electronic system design methods and techniques - EDA technology, students can develop integrated electronic system design professional and necessary vocational skills.

\section{Conclusion}

With the continuous development of information processing technology and continue improving of EDA software design tools, it greatly improved the efficiency and maneuverability of electronic circuit design, and greatly reduce the labor intensity of the designer, so as to bring the revolution for electronic systems design. Because the EDA simulation technology is advanced, practical and versatile, it will lead the future development direction of experimental teaching and play a role to promote the reform the experimental teaching methods and means.

\section{References}

[1] Chunyan Gong: People Education, Vol. 3 (2008) No 53, p.25-26

[2] Huijun Wu: Education Modernization, Vol. 12 (2005) No 27, p.74-76

[3] Zhili Chen: China Education, Vol. 1 (2006) No 33, p.11-14

[4] Shide Zhang: Journal of Electrical \& Electronic Education, Vol. 3 (2007) No33, p.121-124

[5] Long Kong: China Science and Technology Information, Vol. 1 (2006) No 33, p.11-14 